\title{
CHARACTERIZATION OF HYPERSURFACE SINGULARITIES IN POSITIVE CHARACTERISTIC
}

\author{
AMIR SHEHZAD, MUHAMMAD AHSAN BINYAMIN, AND HASAN MAHMOOD
}

\begin{abstract}
The classification of right unimodal and bimodal hypersurface singularities over a field of positive characteristic was given by H. D. Nguyen. The classification is described in the style of Arnold and not in an algorithmic way. This classification was characterized by M. A. Binyamin et al. [Bull. Math. Soc. Sci. Math. Roumanie (N.S.) 61(109) (2018), no. 3, 333-343] for the case when the corank of hypersurface singularities is $\leq 2$. The aim of this article is to characterize the right unimodal and bimodal hypersurface singularities of corank 3 in an algorithmic way by means of easily computable invariants such as the multiplicity, the Milnor number of the given equation, and its blowing-up. On the basis of this characterization we implement an algorithm to compute the type of the right unimodal and bimodal hypersurface singularities without computing the normal form in the computer algebra system Singular.
\end{abstract}

\section{INTRODUCTION}

Let $K\left[\left[x_{1}, \ldots, x_{n}\right]\right]$ be the local ring of formal power series in $n$ variables, $\mathfrak{m}$ its maximal ideal, $K$ an algebraically closed field of characteristic $p>0$, and $\mathcal{R}=\operatorname{Aut}_{K}\left(K\left[\left[x_{1}, \ldots, x_{n}\right]\right]\right)$, the set of all $K$-automorphisms of $K\left[\left[x_{1}, \ldots, x_{n}\right]\right]$. Let $f$ and $g \in \mathfrak{m}$. $f$ is said to be right equivalent to $g, f \sim_{r} g$, if there exists an automorphism $\phi \in \mathcal{R}$ such that $\phi(f)=g$. In case of two (resp. three) variables, we will later use $K[[x, y]]$ (resp. $K[[x, y, z]])$ instead of $K\left[\left[x_{1}, x_{2}\right]\right]$ (resp. $\left.K\left[\left[x_{1}, x_{2}, x_{3}\right]\right]\right)$.

Arnold introduced in the seventies [2, 3, 4] the notion of modality in singularity theory for real and complex singularities. He classified simple, unimodal, and bimodal hypersurface singularities with respect to right equivalence. These are also classifications with respect to contact equivalence. Simple hypersurface singularities in charateristic $p>0$ were classified by Greuel and Kröning [7] with respect to contact equivalence. Greuel and Nguyen [8] classified the simple hypersurface singularities in characteristic $p>0$ with respect to right equivalence. These classifications are characterized in [1].

2010 Mathematics Subject Classification. 14H20, 14 Q05.

Key words and phrases. Right equivalence; Milnor number; Blowing-up.

The research of the second author is supported by the Higher Education Commission of Pakistan under project no. 5688/Punjab/NRPU/R\&D/HEC/2016. 
Nguyen gave the classification of right unimodal and bimodal hypersurface singularities in positive characteristic [10]. In this article we use the results of [10] in order to characterize this classification for unimodal and bimodal hypersurface singularities of corank 3 in terms of certain invariants. We use the names of the singularities from [10, where normal forms are given. In some cases we use blowing-up as a tool to differentiate certain types. We use the right-modality as defined in 8 and used in [10]:

Given $f \in K\left[\left[x_{1}, \ldots, x_{n}\right]\right]$, the family $f_{\lambda}=f+\sum_{i=1}^{N} \lambda_{i} m_{i}$, with $\left\{m_{1}, \ldots, m_{N}\right\}$ being a monomial basis of $m / m\left(\frac{\partial f}{\partial x_{1}}, \ldots, \frac{\partial f}{\partial x_{n}}\right)$, is a semi-universal unfolding of $f$, $\lambda=\left(\lambda_{1}, \ldots, \lambda_{N}\right)$. If the set of singularities $f_{\lambda} \in K\left[\left[x_{1}, \ldots, x_{n}\right]\right], \lambda$ in some Zariski neighbourhood of $0 \in K^{N}$, falls into finitely many families of right equivalence classes depending on $r$ parameters, then $f$ is called right $r$-modal. If $r=1$ (resp. $r=2$ ), then $f$ is called unimodal (resp. bimodal).

\begin{tabular}{llc}
\hline Type & Normal form & modality \\
\hline$P_{8}=T_{3,3,3}$ & $x^{3}+y^{3}+z^{3}+a x y z$ & 1 \\
\hline$Q_{10}$ & $x^{3}+y^{4}+y z^{2}+a x y^{3}$ & 1 \\
\hline$Q_{11}$ & $x^{3}+y z^{2}+x z^{3}+a z^{5}$ & 1 \\
\hline$Q_{12}$ & $x^{3}+y^{5}+y z^{2}+a x y^{4}$ & 1 \\
\hline$S_{11}$ & $x^{4}+y^{2} z+x z^{2}+a x^{3} z$ & 1 \\
\hline$S_{12}$ & $x^{2} y+y^{2} z+x z^{3}+a z^{5}$ & 1 \\
\hline$T_{r, s, t}$ & $x^{r}+y^{s}+z^{t}+a x y z$ & 1 \\
\hline$U_{12}$ & $x^{3}+x z^{2}+y^{4}+a x y z^{2}$ & 1 \\
\hline
\end{tabular}

Table 1: Unimodal hypersurface singularities of corank 3

\section{Characterization of hypersurface Singularities of CORANK 3}

In this section we characterize all right unimodal and bimodal hypersurface singularities of corank 3 in terms of multiplicity, Milnor number, and blowing-ups. We have only to consider the cases $m(f)=2$ and $m(f)=3$, since $f$ is not uni or bimodal if $m(f) \geq 4$ or $\operatorname{corank}(f)>3$ (see Theorems 120 and 121 in [10]). Moreover, we only need to consider $p \geq 5$, because for $p=2$ and $p=3$ there is no unimodal or bimodal hypersurface singularity of corank 3 (see Theorem 136 and Theorems 147 to 151 in [10]).

Proposition 2.1. Let $f \in K[[x, y, z]]$ and $m(f)=2$. Then $f$ is right equivalent to $g(x, y)+z^{2}$ and the type of $f$ is determined by $g$.

Proof. Using the splitting lemma, we obtain $f \sim_{r} g(x, y)+z^{2}$. The type of $f$ can be found in [9, Table 7] and can be determined by using the results of [5].

Proposition 2.2. Let $f \in K[[x, y, z]]$ be such that $m(f)=3$. Then $j^{3}(f)$ is right equivalent to one of the types given in Table 3. 


\begin{tabular}{lll}
\hline Type & Normal form & modality \\
\hline$Q_{16}$ & $x^{3}+y z^{2}+y^{7}+a x y^{5}+b x y^{6}$ & 2 \\
\hline$Q_{17}$ & $x^{3}+y z^{2}+x y^{5}+a y^{8}+b y^{9}$ & 2 \\
\hline$Q_{18}$ & $x^{3}+y z^{2}+y^{8}+a x y^{6}+b x y^{7}$ & 2 \\
\hline$Q_{2,0}$ & $x^{3}+y z^{2}+a x^{2} y^{2}+b x^{2} y^{3}+x y^{4}$ & 2 \\
\hline$Q_{2, q}$ & $x^{3}+y z^{2}+x^{2} y^{2}+a y^{6+q}+b y^{7+q}$ & 2 \\
\hline$S_{16}$ & $x^{2} z+y z^{2}+x y^{4}+a y^{6}+b y^{7}$ & 2 \\
\hline$S_{17}$ & $x^{2} z+y z^{2}+y^{6}+a z y^{4}+b z y^{5}$ & 2 \\
\hline$S_{1,0}$ & $x^{2} z+y z^{2}+y^{5}+a z y^{3}+b z y^{4}$ & 2 \\
\hline$S_{1, q}$ & $x^{2} z+y z^{2}+x^{2} y^{2}+a y^{5+q}+b y^{6+q}$ & 2 \\
\hline$S_{1,2 q-1}^{\sharp}$ & $x^{2} z+y z^{2}+z y^{3}+a x y^{3+q}+b x y^{4+q}$ & 2 \\
\hline$S_{1,2 q}^{\sharp}$ & $x^{2} z+y z^{2}+z y^{3}+a x^{2} y^{2+q}+b x^{2} y^{3+q}$ & 2 \\
\hline$T_{r, s, t}$ & $x^{r}+y^{s}+z^{t}+a x y z+b z^{p}$ & 2 \\
\hline$U_{16}$ & $x^{3}+x z^{2}+y^{5}+a x^{2} y^{2}+a x^{2} y^{3}$ & 2 \\
\hline$U_{1,0}$ & $x^{3}+x z^{2}++x y^{3}+a y^{3} z+b y^{4} z$ & 2 \\
\hline$U_{1,2 q-1}$ & $x^{3}+x z^{2}+x y^{3}+a y^{1+q} z^{2}+b y^{2+q} z^{2}$ & 2 \\
\hline$U_{1,2 q}$ & $x^{3}+x z^{2}+x y^{3}+a y^{3+q} z+b y^{4+q} z$ & 2 \\
\hline
\end{tabular}

Table 2: Bimodal hypersurface singularities of corank 3

\begin{tabular}{ll}
\hline Normal form of $j^{3}(f)$ & Type \\
\hline$x y z$ & I \\
\hline$x^{3}+x y z$ & II \\
\hline$x^{3}+y^{3}+x y z$ & III \\
\hline$x^{3}+y^{3}+z^{3}+a x y z$ & IV \\
\hline$x^{3}+y z^{2}$ & V \\
\hline$x^{2} z+y z^{2}$ & VI \\
\hline$x^{3}+x z^{2}$ & VII \\
\hline$x^{2} y$ & VIII \\
\hline$x^{3}$ & IX \\
\hline
\end{tabular}

Table 3: Classification of three jet of $f \in K[[x, y, z]]$

Proof. The result follows from Theorems 74 to 119 in [10]. 
Remark 2.3. From Theorem 118 and Theorem 119 in [10, it follows that if $j^{3}(f) \sim_{r} x^{2} y$ or $j^{3}(f) \sim_{r} x^{3}$ then $\operatorname{rmod}(f) \geq 3$. Therefore we only need to discuss the rest of the seven possibilities (I-VII) of $j^{3}(f)$.

Lemma 2.4. Let $g=x y z+\sum_{t \geq q} a_{t} x^{t}+\sum_{u \geq r} b_{u} y^{u}+\sum_{v \geq s} c_{v} z^{v}$ with $q \leq r \leq s$. Then $q, r$, and $s$ can be computed by using blowing-ups.

Proof. Consider the blowing-up in the first chart defined by $x \rightarrow x, y \rightarrow x y$, and $z \rightarrow x z$; then we have

$$
g(x, x y, x z)=x^{3} y z+\sum_{t \geq q} a_{t} x^{t}+\sum_{u \geq r} b_{u}(x y)^{u}+\sum_{v \geq s} c_{v}(x z)^{v} .
$$

The strict transform is

$$
\frac{g(x, x y, x z)}{x^{3}}=y z+\sum_{t \geq q} a_{t} x^{t-3}+\sum_{u \geq r} b_{u} x^{u-3} y^{u}+\sum_{v \geq s} c_{v} x^{v-3} z^{v} .
$$

Then obviously the Milnor number of $\frac{g(x, x y, x z)}{x^{3}}$ is $q-4$. Similarly we obtain $r-4$ and $s-4$ from the other charts.

Lemma 2.5. Let $f \in K[[x, y, z]]$ be such that $m(f)=3$. If the zero-set $V\left(j^{3}(f)\right)$ of $j^{3}(f)$ is the intersection of three planes and has as singular locus the union of three lines $V(x, y) \cup V(x, z) \cup V(y, z)$, then $f \sim_{r} x y z+\sum_{t \geq q} a_{t} x^{t}+\sum_{u \geq r} b_{u} y^{u}+\sum_{v \geq s} c_{v} z^{v}$ with $q \leq r \leq s$ and $a_{q} \neq 0, b_{r} \neq 0, c_{s} \neq 0$. Moreover, the triple $(q, r, s)$ modulo permutation is an invariant under right equivalence for this type of singularities.

Proof. If the zero-set $V\left(j^{3}(f)\right)$ is the intersection of three planes ${ }^{*}$ and has as singular locus the union of three lines $V(x, y) \cup V(x, z) \cup V(y, z)$, then we can assume that

$$
f \sim_{r} g=x y z+\sum_{i+j+k \geq 4} a_{i, j, k} x^{i} y^{j} z^{k} .
$$

To prove the statement we use induction on $m$. We write $g=x y z+\alpha_{1}(x)+\beta_{1}(y)+$ $\gamma_{1}(z)+g_{0}(x, y, z) \bmod \mathfrak{m}^{m+1}$, where $m(\alpha), m(\beta), m(\gamma) \geq 4$ and $g_{0}$ is a homogeneous polynomial of degree $m \geq 4$ with $g_{0}(x, 0,0)=g_{0}(0,0, z)=g_{0}(0, y, 0)=0$.

Write $g_{0}(x, y, z)=y z h_{0}(y, z)+x z h_{1}(x, z)+x y h_{2}(x, y, z)$. After using the transformations

$$
x \rightarrow x-h_{0}, \quad y \rightarrow y-h_{1}, \quad z \rightarrow z-h_{2}
$$

we get $f \sim_{r} x y z+\alpha(x)+\beta(y)+\gamma(z) \bmod \mathfrak{m}^{m+1}$. Iterating this we obtain

$$
f \sim_{r} x y z+\sum_{t \geq q} a_{t} x^{t}+\sum_{u \geq r} b_{u} y^{u}+\sum_{v \geq s} c_{v} z^{v}
$$

with $q \leq r \leq s$.

To prove the invariance of $(q, r, s)$ modulo permutation, we assume that $f=$ $x y z+$ terms of order $\geq 4 \sim_{r} g=x y z+$ terms of order $\geq 4$, i.e. there exists an automorphism $\varphi: K[[x, y, z]] \rightarrow K[[x, y, z]]$ such that $\varphi(f)=g$. This implies that $\varphi(x y z)=x y z \bmod (x, y, z)^{4}$, i.e. the linear part of $\varphi$ is a permutation of the

${ }^{*}$ This means that, after a suitable linear coordinate change, $j^{3}(f)=x y z$. 
variables. Since we have to prove the invariance of $(q, r, s)$ modulo permutation, we may assume that $\varphi=i d \bmod (x, y, z)^{4}$. This implies that in the charts the Milnor number of the blowing-up of $f$ and $g$ is the same. Using Lemma 2.4, the result follows.

Proposition 2.6. Let $f \in K[[x, y, z]]$ be such that $m(f)=3$. If the zero-set $V\left(j^{3}(f)\right)$ is the intersection of three planes and has as singular locus the intersection of three lines $V(x, y) \cup V(x, z) \cup V(y, z)$, then

(1) if $s<p$ then $f$ is unimodal of type $T_{q, r, s}$;

(2) if $r<p \leq s<2 p$ then $f$ is bimodal of type $T_{q, r, s}$;

(3) if $s \geq 2 p$ then $\operatorname{rmod}(f) \geq 3$,

where $(q, r, s)$ is defined as in Lemma 2.5

Proof. If the zero-set $V\left(j^{3}(f)\right)$ is the intersection of three planes and has as a singular locus the intersection of three lines $V(x, y) \cup V(x, z) \cup V(y, z)$, then by Lemma 2.5

$$
f \sim_{r} g=x y z+\sum_{t \geq q} a_{t} x^{t}+\sum_{u \geq r} b_{u} y^{u}+\sum_{v \geq s} c_{v} z^{v},
$$

with $q \leq r \leq s$. Moreover, by using Lemma 2.4 we can compute $q, r, s$ and hence the type of $f$.

Lemma 2.7. Let $f \in K[[x, y, z]]$ be such that $m(f)=3$. If the zero-set $V\left(j^{3}(f)\right)$ is the intersection of a plane and a node and has as singular locus the union of the lines $V(x, y) \cup V(x, z)$ and the point $V(x, y, z)$ as embedded point, then $f \sim_{r}$ $x y z+x^{3}+\sum_{u \geq r} b_{u} y^{u}+\sum_{v \geq s} c_{v} z^{v}$, with $4 \leq r \leq s$ and $b_{r} \neq 0, c_{s} \neq 0$. Moreover, the pair $(r, s)$ modulo permutation is an invariant under right equivalence for this type of singularities.

Proof. Since the zero-set $V\left(j^{3}(f)\right)$ is the intersection of a plane and a node and has as singular locus the union of the lines $V(x, y) \cup V(x, z)$ and the point $V(x, y, z)$ as embedded point, we can assume that $f \sim_{r} g=x y z+x^{3}+\sum_{i+j+k \geq 4} a_{i, j, k} x^{i} y^{j} z^{k}$.

Similarly to the previous case, we use induction on $m$. We write $g=x y z+x^{3}+$ $\alpha(x)+\beta(y)+\gamma(z)+g_{0}(x, y, z) \bmod \mathfrak{m}^{m+1}$, where $m(\alpha), m(\beta), m(\gamma) \geq 4$ and $g_{0}$ is a homogeneous polynomial of degree $m \geq 4$ with $g_{0}(0,0, z)=0=g_{0}(0, y, 0)$.

Write $g_{0}(x, y, z)=y z h_{0}(y, z)+k(x, y, z)$. After using the transformation

$$
x \rightarrow x-h_{0}-\frac{a_{m, 0,0}}{3} x^{m-2}, \quad y \rightarrow y, \quad z \rightarrow z
$$

we get $g\left(x-h_{0}-\frac{a_{m, 0,0}}{3} x^{m-2}, y, z\right)=x y z+x^{3}+\beta_{1}(y)+\gamma_{1}(z)+g_{1}(x, y, z) \bmod \mathfrak{m}^{m+1}$, where $g_{1}$ is either zero or homogeneous of degree $m$ with $g_{0}(0, y, z)$. If $g_{1} \neq 0$ then write

$$
g_{1}(x, y, z)=x z h_{1}(x, z)+x y h_{2}(x, y, z) .
$$

Now by using the transformation

$$
x \rightarrow x, \quad y \rightarrow y-h_{1}, \quad z \rightarrow h_{2}
$$


we get $f \sim_{r} x y z+x^{3}+\beta_{1}(y)+\gamma_{1}(z) \bmod \mathfrak{m}^{m+1}$. Using induction we obtain

$$
f \sim_{r} x y z+x^{3}+\sum_{u \geq r} b_{u} y^{u}+\sum_{v \geq s} c_{v} z^{v}
$$

with $4 \leq r \leq s$.

The proof of invariance of $(r, s)$ is similar to the corresponding proof of Lemma 2.5

Lemma 2.8. Let $g=x y z+x^{3}+\sum_{u \geq r} b_{u} y^{u}+\sum_{v \geq s} c_{v} z^{v}$ with $4 \leq r \leq s$. Then $r$ and $s$ can be computed by using blowing-ups.

Proof. The proof is similar to that of Lemma 2.4

Proposition 2.9. Let $f \in K[[x, y, z]]$ be such that $m(f)=3$. If the zero-set $V\left(j^{3}(f)\right)$ is the intersection of a plane and a node and has as singular locus the union of the lines $V(x, y) \cup V(x, z)$ and the point $V(x, y, z)$ as embedded point, then

(1) if $s<p$ then $f$ is unimodal of type $T_{3, r, s}$;

(2) if if $r<p \leq s<2 p$ then $f$ is bimodal of type $T_{3, r, s}$;

(3) if $s \geq 2 p$ then $\operatorname{rmod}(f) \geq 3$,

where $(r, s)$ is defined as in Lemma 2.7 .

Proof. Since the zero-set $V\left(j^{3}(f)\right)$ is the intersection of a plane and a node and has as singular locus the union of the lines $V(x, y) \cup V(x, z)$ and the point $V(x, y, z)$ as embedded point, we have by Lemma 2.7 that

$$
f \sim_{r} g=x y z+x^{3}+\sum_{u \geq r} b_{u} y^{u}+\sum_{v \geq s} c_{v} z^{v}
$$

with $4 \leq r \leq s$. Moreover, by using Lemma 2.8 we can compute $r, s$ and hence the type of $f$.

Lemma 2.10. If the zero-set $V\left(j^{3}(f)\right)$ is irreducible and has in the singular locus a fat point of multiplicity 6 , then $f \sim_{r} x y z+x^{3}+y^{3}+\sum_{v \geq s} c_{v} z^{v}$ with $4 \leq s$ and $c_{s} \neq 0$.

Proof. Since the zero-set $V\left(j^{3}(f)\right)$ is irreducible and has in the singular locus a fat point of multiplicity 6 , we have $f \sim_{r} g=x y z+x^{3}+y^{3}+\sum_{i+j+k \geq 4} a_{i, j, k} x^{i} y^{j} z^{k}$.

We write $g=x y z+x^{3}+y^{3}+\alpha(x)+\beta(y)+\gamma(z)+g_{0}(x, y, z) \bmod \mathfrak{m}^{m+1}$, where $m(\alpha), m(\beta), m(\gamma) \geq 4$ and $g_{0}$ is a homogeneous polynomial of degree $m \geq 4$ with $g_{0}(x, 0,0)=g_{0}(0,0, z)=g_{0}(0, y, 0)=0$.

Write $g_{0}(x, y, z)=y z h_{0}(y, z)+x z h_{1}(x, z)+g_{1}(x, y, z)$. After using the transformation

$$
\begin{aligned}
& x \rightarrow x-h_{0}-\frac{a_{m, 0,0}}{3} x^{m-2}+3 a_{1,0, m-1} y z^{m-3}, \\
& y \rightarrow y-h_{1}-\frac{a_{0, m, 0}}{3} y^{m-2}+3 a_{0,1, m-1} x z^{m-3}, \\
& z \rightarrow z
\end{aligned}
$$


$g$ transforms into $x y z+x^{3}+y^{3}+\gamma_{1}(z)+g_{2}(x, y, z) \bmod \mathfrak{m}^{m+1}$, where $g_{2}$ is either zero or homogeneous of degree $m$. If $g_{2} \neq 0$ then write

$$
g_{2}(x, y, z)=x y k_{2}(x, y, z) \text {. }
$$

After using the transformation

$$
x \rightarrow x, \quad y \rightarrow y, \quad z \rightarrow z-k_{2}
$$

we get $f \sim_{r} x y z+x^{3}+y^{3}+\gamma_{1}(z) \bmod \mathfrak{m}^{m+1}$. Using induction on $m$ we obtain

$$
f \sim_{r} x y z+x^{3}+y^{3}+\sum_{v \geq s} c_{v} z^{v}
$$

with $4 \leq s$.

Lemma 2.11. Let $g=x y z+x^{3}+y^{3}+\sum_{v \geq s} c_{v} z^{v}$. Then $s$ can be computed by using blowing-ups.

Proof. The proof is similar to that of Lemma 2.4

Proposition 2.12. Let $f \in K[[x, y, z]]$ be such that $m(f)=3$. If the zero-set $V\left(j^{3}(f)\right)$ is irreducible and has in the singular locus a fat point of multiplicity 6 , then

(1) if $s<p$ then $f$ is unimodal of type $T_{3,3, s}$;

(2) if $p \leq s<2 p$ then $f$ is bimodal of type $T_{3,3, s}$;

(3) if $s \geq 2 p$ then $\operatorname{rmod}(f) \geq 3$,

where $s$ is the smallest exponent of $z$, as appeared in Lemma 2.10.

Proof. Since the zero-set $V\left(j^{3}(f)\right)$ is irreducible and has in the singular locus a fat point of multiplicity 6 , we have by Lemma 2.7

$$
f \sim_{r} g=x y z+x^{3}+y^{3}+\sum_{v \geq s} c_{v} z^{v}
$$

with $4 \leq s$. Moreover, by using the Lemma 2.11 we can compute $s$ and hence the type of $f$.

Proposition 2.13. Let $f \in K[[x, y, z]]$ be such that $m(f)=3$. If $\mu\left(j^{3}(f)\right)=8$ then $f$ is of type $T_{3,3,3}$.

Proof. The result follows from step 75 in the singularity determinator of [10].

Definition 2.14. Let $\left\{\alpha_{i}\right\}$ be a system of $n$ points defining an affine hyperplane $H$ in $\mathbb{R}^{n}$ and let $v: \mathbb{R}^{n} \rightarrow \mathbb{R}$ be the linear form defining $H$ with $v\left(\alpha_{i}\right)=1$ for all $i$. The quasijet of $f$ determined by $\left\{X^{\alpha_{i}}\right\}$, denoted by $j_{\left\{X^{\left.\alpha_{i}\right\}}\right.}(f)$, is the image of $f$ in $K[[X]]$ modulo the ideal generated by $X^{\alpha}, v(\alpha)>1$.

Proposition 2.15. Let $f \in K[[x, y, z]]$ be such that $m(f)=3$. Then $\operatorname{rmod}(f) \geq 3$ in the following cases:

(1) the zero-set $V\left(j^{3}(f)\right)$ is irreducible and has in the singular locus a fat point of multiplicity 4 and $j_{x^{3}, y z^{2}, y^{6}}(f)=x^{3}+y z^{2}+x^{2} y^{2}$ and $\mu(f) \geq p+5$; 
(2) the zero-set $V\left(j^{3}(f)\right)$ is the intersection of a plane and a node and has as singular locus the line $V(x, z)$ and $j_{x^{2} y, y z^{2}, y^{5}}(f)=x^{2} z+y z^{2}+x^{2} y^{2}$ and $\mu(f) \geq p+9$

(3) the zero-set $V\left(j^{3}(f)\right)$ is the intersection of a plane and a node and has as singular locus the line $V(x, z)$ and $j_{x^{2} y, y z^{2}, y^{5}}(f)=x^{2} z+y z^{2}+z y^{3}$ and $\mu(f) \geq p+9$

(4) the zero-set $V\left(j^{3}(f)\right)$ is the intersection of a plane and a node and has as singular locus the line $V(x, z)$ and $j_{x^{2} y, y z^{2}, y^{6}}(f)=x^{2} z+y z^{2}$;

(5) the zero-set $V\left(j^{3}(f)\right)$ is the intersection of three planes and has as singular locus the line $V(x, z)$ and $j_{x^{3}, z^{3}, x y^{3}}(f)=x^{3}+x z^{2}+x y^{3}$ and $\mu(f) \geq p+13$;

(6) the zero-set $V\left(j^{3}(f)\right)$ is the intersection of three planes and has as singular locus the line $V(x, z)$ and $j_{x^{3}, z^{3}, x y^{3}}(f)=x^{3}+x z^{2}$;

(7) $j^{3}(f)$ has two linear factors, one of multiplicity 1 and one of multiplicity 2 ;

(8) $j^{3}(f)$ has only one linear factor of multiplicity 3 .

Proof. (1) is a consequence of step 90; (2) is a consequence of step 99; (3) is a consequence of step 101; (4) is a consequence of step 105; (5) is a consequence of step 113; (6) is a consequence of step 117; (7) is a consequence of step 118; and (8) is a consequence of step 119 in the singularity determinator of [10].

Notation. Consider the charts of the blowing-up given by

$$
\begin{aligned}
& 1: x \rightarrow x, y \rightarrow x y, z \rightarrow x z, \\
& 2: x \rightarrow x y, y \rightarrow y, z \rightarrow y z, \\
& 3: x \rightarrow x z, y \rightarrow y z, z \rightarrow z .
\end{aligned}
$$

By $j^{k}\left(f_{i, n}\right)$ we denote the $k$-th jet of the strict transformation of the $n$-th blowingup corresponding to the $i$-th chart.

Proposition 2.16. Let $f \in K[[x, y, z]]$ be such that $m(f)=3$. If the zero-set $V\left(j^{3}(f)\right)$ is irreducible and has in the singular locus a fat point of multiplicity 4 , then

(1) if $10 \leq \mu(f) \leq 12$ then $f$ is unimodal of type $Q_{\mu(f)}$;

(2) if $\mu(f)=14,15$ then $f$ is bimodal of type $Q_{2, \mu(f)-14}$;

(3) if $16 \leq \mu(f) \leq 18$ then $f$ is bimodal of type $Q_{\mu(f)}$ or $Q_{2, \mu(f)-14}$;

(4) if $19 \leq \mu(f)$ then $f$ is bimodal of type $Q_{2, \mu(f)-14}$.

Proof. We may assume that $f=x^{3}+y z^{2}+\sum_{i+j+k \geq 4} a_{i, j, k} x^{i} y^{j} z^{k}$. The analysis of Theorem 81 to Theorem 89 in [10] gives that $f$ is unimodal of type $Q_{\mu(f)}$ if $10 \leq \mu(f) \leq 12, f$ is bimodal of type $Q_{2, \mu(f)-14}$ if $\mu(f)=14,15$ or $19 \leq \mu(f)$, and if $16 \leq \mu(f) \leq 18$ then $f$ is bimodal of type $Q_{\mu(f)}$ or $Q_{2, \mu(f)-14}$. These three cases can be differentiated by using blowing-ups as follows.

If $\mu(f)=16$, then if $V\left(j^{2}\left(f_{2,2}\right)\right)$ is the intersection of two planes then $f$ is of type $Q_{16}$ and if $V\left(j^{2}\left(f_{2,2}\right)\right)$ is a double plane then $f$ is of type $Q_{2,2}$. If $\mu(f)=17$, then if $f_{2,3}$ is a smooth curve then $f$ is of type $Q_{17}$ and if $V\left(j^{2}\left(f_{2,3}\right)\right)$ is the intersection of two planes then $f$ is of type $Q_{2,3}$. If $\mu(f)=18$, then if $f_{2,3}$ is a smooth curve then $f$ is of type $Q_{18}$ and if $V\left(j^{2}\left(f_{2,3}\right)\right)$ is a double plane then $f$ is of type $Q_{2,4}$. 
Proposition 2.17. Let $f \in K[[x, y, z]]$ be such that $m(f)=3$. If the zero-set $V\left(j^{3}(f)\right)$ is the intersection of a plane and a node and has as singular locus the line $V(x, z)$, then

(1) if $\mu(f)=11,12$ then $f$ is unimodal of type $S_{\mu(f)}$;

(2) if $\mu(f)=14$ then $f$ is bimodal of type $S_{1,0}$;

(3) if $\mu(f)=15$ then $f$ is bimodal of type $S_{1,1}$ or $S_{1,1}^{\sharp}$;

(4) if $\mu(f)=16$ then $f$ is bimodal of type $S_{16}$ or $S_{1,2}$ or $S_{1,2}^{\sharp}$;

(5) if $\mu(f)=17$ then $f$ is bimodal of type $S_{17}$ or $S_{1,3}$ or $S_{1,3}^{\sharp}$;

(6) if $16<\mu(f)<2 p+8$ and $\mu(f)$ is even then $f$ is bimodal of type $S_{1, \mu(f)-14}$ or $S_{1, \mu(f)-13}^{\sharp}$;

(7) if $19 \leq \mu(f)<2 p+11$ and $\mu(f)$ is odd then $f$ is bimodal of type $S_{1, \mu(f)-14}$ or $S_{1, \mu(f)-14}^{\sharp}$.

Proof. We may assume that $f=x^{2} z+y z^{2}+\sum_{i+j+k \geq 4} a_{i, j, k} x^{i} y^{j} z^{k}$. The analysis of Theorem 92 to Theorem 105 in [10] gives that if $\mu(f)=11,12$ then $f$ is unimodal of type $S_{\mu(f)}$ and if $\mu(f)=14$ then $f$ is bimodal of type $S_{1,0}$. If $\mu(f)=15$, then if $V\left(j^{2}\left(f_{2,1}\right)\right)$ is a double plane then $f$ is of type $S_{1,1}$ and if $V\left(j^{2}\left(f_{2,1}\right)\right)$ is the intersection of two planes then $f$ is of type $S_{1,1}^{\sharp}$. If $\mu(f)=16$, then if $V\left(j^{2}\left(f_{2,1}\right)\right)$ is the intersection of two planes then $f$ is of type $S_{1,2}^{\sharp}$ and if $V\left(j^{2}\left(f_{2,1}\right)\right)$ is a double plane and $V\left(j^{2}\left(f_{2,2}\right)\right)$ is the intersection of two planes then $f$ is bimodal of type $S_{1,2}$ and if $V\left(j^{2}\left(f_{2,1}\right)\right)$ is a double plane and $V\left(j^{2}\left(f_{2,2}\right)\right)$ is a smooth curve then $f$ is bimodal of type $S_{16}$. If $\mu(f)=17$, then if $V\left(j^{2}\left(f_{2,1}\right)\right)$ is the intersection of two planes then $f$ is of type $S_{1,3}^{\sharp}$ and if $V\left(j^{2}\left(f_{2,1}\right)\right)$ and $V\left(j^{2}\left(f_{2,2}\right)\right)$ is a double plane then $f$ is bimodal of type $S_{1,3}$ and if $V\left(j^{2}\left(f_{2,1}\right)\right)$ is a double plane and $f_{2,2}$ is a smooth curve then $f$ is bimodal of type $S_{17}$. Now if $16<\mu(f)<2 p+8$ and $\mu(f)$ is even, then if $V\left(j^{2}\left(f_{2,1}\right)\right)$ is a double plane then $f$ is bimodal of type $S_{1, \mu(f)-14}$ and if $V\left(j^{2}\left(f_{2,1}\right)\right)$ is the intersection of two planes then $f$ is of type $S_{1, \mu(f)-13}^{\sharp}$. Similarly, if $19 \leq \mu(f)<2 p+11$ and $\mu(f)$ is odd, then if $V\left(j^{2}\left(f_{2,1}\right)\right)$ is a double plane then $f$ is bimodal of type $S_{1, \mu(f)-14}$ and if $V\left(j^{2}\left(f_{2,1}\right)\right)$ is the intersection of two planes then $f$ is of type $S_{1, \mu(f)-14}^{\sharp}$.

Proposition 2.18. Let $f \in K[[x, y, z]]$ be such that $m(f)=3$. If the zero-set $V\left(j^{3}(f)\right)$ is the intersection of three planes and has as singular locus the line $V(x, z)$, then

(1) if $\mu(f)=12$ then $f$ is unimodal of type $U_{12}$;

(2) if $\mu(f)=14$ then $f$ is bimodal of type $U_{1,0}$;

(3) if $\mu(f)=16$ then $f$ is bimodal of type $U_{16}$ or $U_{1,1}$;

(4) if $15 \leq \mu(f)<2 p+11$ and $\mu(f)$ is odd then $f$ is bimodal of type $U_{1, \frac{\mu(f)-13}{2}}$;

(5) if $16<\mu(f)<2 p+8$ and $\mu(f)$ is even then $f$ is bimodal of type $U_{1, \frac{\mu(f)-14}{2}}$.

Proof. We may assume that $f=x^{3}+x z^{2}+\sum_{i+j+k \geq 4} a_{i, j, k} x^{i} y^{j} z^{k}$. The analysis of Theorem 107 to Theorem 112 in [10] gives that $f$ is unimodal of type $U_{12}$ if 
$\mu(f)=12 ; f$ is bimodal of type $U_{1,0}$ if $\mu(f)=14 ; f$ is bimodal of type $U_{1, \frac{\mu(f)-13}{2}}$ if $15 \leq \mu(f)<2 p+11$ and $\mu(f)$ is odd; $f$ is bimodal of type $U_{1, \frac{\mu(f)-14}{2}}$ if $16<$ $\mu(f)<2 p+8$ and $\mu(f)$ is even; and $f$ is bimodal of type $U_{16}$ or $U_{1,1}$ if $\mu(f)=16$. $U_{16}$ and $U_{1,1}$ can be differentiated by using blowing-ups such that if $V\left(j^{2}\left(f_{2,1}\right)\right)$ is the intersection of two planes then $f$ is of type $U_{1,1}$ and if $V\left(j^{2}\left(f_{2,1}\right)\right)$ is a double plane then $f$ is of type $U_{16}$.

\section{SHORT DESCRIPTION OF THE CLASSIFIER}

In this section we give the description of our classifier and its implementation in Singular. This classifier is used for computing the type of the unimodal and bimodal hypersurface singularities in characteristic $p>0$ with respect to right equivalence. Our classifier is based on the algorithm classiFy (f), which classifies hypersurface singularities of modality one and two of corank 3 with respect to right equivalence.

We consider $p \geq 5, n=$ number of variables $=3$, and $\operatorname{corank}(f)=3$. If $\mu(f)$, the Milnor number of $f$, is finite, then our classifier checks the multiplicity of $f$. If $m(f)=2$ then the classifier transforms $f$ into $g(x, y)+z^{2}$ (by using the splitting lemma). At this stage our classifier determines the type of $g^{f}$ and then returns the corresponding type of $f$.

Now if $m(f)=3$ the classifier computes $\mu\left(j^{3}(f)\right)$; if $\mu\left(j^{3}(f)\right)=8$ then it gives the type $T_{3,3,3}$, otherwise the classifier makes some coordinate change and transforms $j^{3}(f)$ into one of $x y z, x y z+x^{3}, x y z+x^{3}+y^{3}, x^{3}+y z^{2}, x^{2} z+y z^{2}$, or $x^{3}+x z^{2}$.

If $j^{3}(f)=x y z$, the classifier makes some coordinate change and transforms $f$ into $x y z+\sum_{t \geq q} a_{t} x^{t}+\sum_{u \geq r} b_{u} y^{u}+\sum_{v \geq s} c_{v} z^{v}$. At this step the classifier performs blow-ups of $f$ at the origin to compute $q, r, s$ and then gives $T_{q, r, s}$.

If $j^{3}(f)=x y z+x^{3}$, the classifier makes some coordinate change and transforms $f$ into $x y z+x^{3}+\sum_{u \geq r} b_{u} y^{u}+\sum_{v \geq s} c_{v} z^{v}$. At this step the classifier performs blow-ups of $f$ at the origin to compute $r, s$ and then gives $T_{3, r, s}$.

If $j^{3}(f)=x y z+x^{3}+y^{3}$, the classifier makes some coordinate change and transforms $f$ into $x y z+x^{3}+y^{3}+\sum_{v \geq s} c_{v} z^{v}$. At this step the classifier performs blow-ups of $f$ at the origin to compute $s$ and then gives $T_{3,3, s}$.

If $j^{3}(f)=x^{3}+y z^{2}$, the classifier gives that $f$ is of type $Q_{\mu(f)}$ or $Q_{2, \mu(f)-14}$. The classifier differentiates these types as explained in Proposition 2.16. Now if $j^{3}(f)=x^{2} z+y z^{2}$, then the classifier gives $f$ is of type $S_{\mu(f)}$ or $S_{1, \mu(f)-14}$ or $S_{1, \mu(f)-13}^{\sharp}$ or $S_{1, \mu(f)-14}^{\sharp}$. The classifier differentiates these types as explained in Proposition 2.17. And if $j^{3}(f)=x^{3}+x z^{2}$, then the classifier gives $f$ is of type $U_{\mu(f)}$ or $U_{1, \frac{\mu(f)-13}{2}}$ or $U_{1, \frac{\mu(f)-14}{2}}$. The classifier differentiates these types as explained in Proposition 2.18

\footnotetext{
${ }^{\dagger}$ The type of $g(x, y)$ can be determined by using the procedure uniBimodalReq $(f)$, implemented in 5 ]
} 
3.1. Singular examples. We have implemented the algorithm in the computer algebra system Singular [6], using version 4-1-2. Code can be downloaded from mathcity.org/junaid. We give some examples:

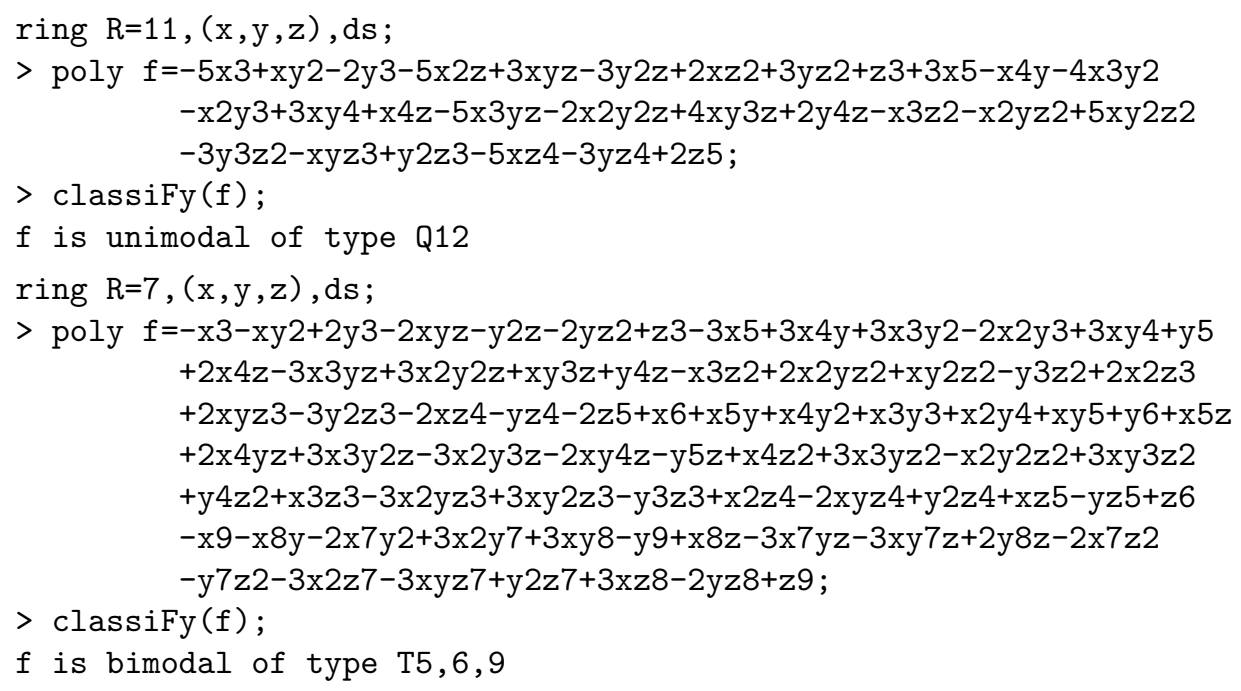

\section{ACKNOWLEDGMENTS}

Authors are thankful to the referee for valuable comments that led to a corrected and improved version of the paper.

\section{REFERENCES}

[1] D. Afzal, M. A. Binyamin and F. K. Janjua, On the classification of simple singularities in positive characteristic, An. Ştiinţ. Univ. "Ovidius" Constanţa Ser. Mat. 22 (2014), no. 2, 5-19. MR 3195690

[2] V. I. Arnol'd, Normal forms for functions near degenerate critical points, the Weyl groups $A_{k}, D_{k}, E_{k}$ and Lagrangian singularities, Funktsional. Anal. i Prilozhen. 6 (1972), no. 4, 3-25; Funct. Anal. Appl. 6 (1972), no. 4, 254-272. MR 0356124

[3] V. I. Arnol'd, Classification of unimodal critical points of functions, Funktsional. Anal. $i$ Prilozhen. 7 (1973), no. 3, 75-76; Funct. Anal. Appl. 7 (1973), no. 3, 230-231. MR 0324079.

[4] V. I. Arnol'd, Local normal forms of functions, Invent. Math. 35 (1976), 87-109. MR 0467795

[5] M. A. Binyamin et al., Classification of right unimodal and bimodal hypersurface singularities in positive characteristic by invariants, Bull. Math. Soc. Sci. Math. Roumanie (N.S.) 61(109) (2018), no. 3, 333-343. MR 3838949

[6] W. Decker et al., Singular 4-1-2 - A computer algebra system for polynomial computations. http://www.singular.uni-kl.de 2019.

[7] G.-M. Greuel and H. Kröning, Simple singularities in positive characteristic, Math. Z. 203 (1990), no. 2, 339-354. MR 1033443 
[8] G.-M. Greuel and H. D. Nguyen, Right simple singularities in positive characteristic, J. Reine Angew. Math. 712 (2016), 81-106. MR 3466548

[9] H. D. Nguyen, Classification of singularities in positive characteristic, doctoral dissertation, Universitët Kaiserslautern, 2013.

[10] H. D. Nguyen, Right unimodal and bimodal singularities in positive characteristic, Int. Math. Res. Not. IMRN 2019, no. 6, 1612-1641. MR 3932590

\author{
Amir Shehzad \\ Department of Mathematics, Government College University, Faisalabad, Pakistan \\ zain. ahmad54@yahoo.com \\ Muhammad Ahsan Binyamin ${ }^{凶}$ \\ Department of Mathematics, Government College University, Faisalabad, Pakistan \\ ahsanbanyamin@gmail.com \\ Hasan Mahmood \\ Department of Mathematics, Government College University, Lahore 54000, Pakistan \\ hasanmahmood@gcu.edu.pk
}

Received: February 3, 2019

Accepted: October 25, 2019 